\title{
Sensing and Classifying Impairments of GPS Reception on Mobile Devices
}

\author{
Henrik Blunck, Mikkel Baun Kjærgaard, and Thomas Skjødeberg Toftegaard \\ Department of Computer Science \\ Aarhus University, Denmark \\ $\{$ blunck, mikkelbk, tst $\} a_{c s} . a u . d k$
}

\begin{abstract}
Positioning using GPS receivers is a primary sensing modality in many areas of pervasive computing. However, previous work has not considered how people's body impacts the availability and accuracy of GPS positioning and for means to sense such impacts. We present results that the GPS performance degradation on modern smart phones for different hand grip styles and body placements can cause signal strength drops as high as 10-16 dB and double the positioning error. Furthermore, existing phone applications designed to help users identify sources of GPS performance impairment are restricted to show raw signal statistics. To help both users as well as application systems in understanding and mitigating body and environment-induced effects, we propose a method for sensing the current sources of GPS reception impairment in terms of body, urban and indoor conditions. We present results that show that the proposed autonomous method can identify and differentiate such sources, and thus also user environments and phone postures, with reasonable accuracy, while relying solely on GPS receiver data as it is available on most modern smart phones.
\end{abstract}

\section{Introduction}

Positioning using GPS receivers is a primary sensing modality in many areas of pervasive computing, such as behavior recognition (e.g., health status monitoring [20]), collaborative sensing (map generation [15] and environment impact monitoring [17]) and community applications (e.g., Micro-Blogging [4] and GeoPages [3]). In these application domains, the GPS receivers are assumed to be worn and used by people during their everyday life. However, the mentioned articles do not consider the impact of the user's body on the positioning performance. Several of the above articles mention that the applications described depend on GPS performance parameters such as availability and accuracy, but link difference and impairment in GPS performance only to the user's surrounding environments, e.g. urban or indoors.

In the first part of our paper, we study and analyze the body impacts on the performance of GPS receivers, focusing on in-phone systems, and intending to inform researchers and developers about these impacts. Our work builds on knowledge from existing studies of the body impact on in-phone GSM communication [119], while our methodology as well as our analysis results differ in nature from those described for GSM communication, since, first, the performance parameters of GPS differ from those of communication services, and, second, since a variety of factors, other than 
body effects, impacts GPS positioning more severely than GSM communication (due to the weakness of the GPS signals); such factors include the user's environment (e.g. urban or indoor) as well as, potentially, other simultaneous phone operations, e.g. GSM or WiFI transmissions or CPU computations [5]. Our study is also motivated by the recent body related issues with modern antenna designs in mobile phones [7] and by a recent short paper by Vaitl et al. [24] who quantify the GPS positioning accuracy for four on-body locations and three phone models in walking experiments.

The second part of this paper is motivated by the fact that existing mobile phone applications designed to help users identify sources of GPS performance degradation are restricted to radar views of satellites' signal strength and accuracy estimates. To help both users as well as application systems in understanding and mitigating body, urban and indoor effects, we want to provide information to the user about which effects are impacting the current performance of the GPS. For the indoor effects we build on the results of our recent study of indoor positioning using GPS presented in Kjærgaard et al. [8]. Consequently, in the second part of this paper we present a concept for how to differentiate these effects utilizing only signal quality data made available by inphone GPS modules, enabling the GPS receiver as a new sensor modality for sensing body placement and environment. Our method calculates a number of features from the signal quality data among others it compares data to an open sky model of how strong signals should have been received given no impairments. The calculated feature values are used as an input to a standard machine learning algorithm that outputs a classification of current positioning impairments.

This concept is motivated foremost by the potential of information about GPS impairments and respective sources to improve GPS positioning quality and quality awareness: Both through GPS receiver algorithms, middleware [14] and application systems, utilizing such information, but also via informing the user directly via on-phone applications about current impairments, increasing his understanding of the position quality and help answering questions, such as "What is impacting my GPS positioning accuracy?" and "Can I improve GPS performance by changing my grip style or placement?" One might consider if the need for answering these questions could be removed by switching to other positioning means, such as WiFi or GSM positioning. But while we found body impacts to cause GPS positioning errors in the range between three to thirty meters, the WiFi or GSM positioning exhibits usually even larger errors in rural and urban areas [13].

We make the following contributions in this work: First, we argue that body related issues are significant for GPS performance and present results for different hand grip styles and body placements which show that signal strength drops as high as 10-16 dB can be experienced and double the positioning error. Finally, we propose a method for sensing and classifying GPS reception and positioning impairments in terms of body, urban and indoor conditions using a set of features calculated via a model for open-sky conditions. We present results that show that the method can estimate the correct cause with reasonable accuracies.

The remainder of this paper is structured as follows: In Section 2 we give a brief introduction and overview of research on GPS with a focus on in-phone GPS systems. In Section 3 we present our study of body-related impacts on GPS reception. In Section 4 
we present the proposed method for sensing present GPS performance impairments and for identifying their sources. In Section 5 we discuss shortcomings and potential improvements and utilizations of the proposed method and provide directions for future work. Finally, Section 6 concludes the paper.

\section{GPS: Operation Basics and Sources of Impairment}

In this section we review basic concepts and recent advances in GPS positioning, as well as, research on how the user's environment impacts GPS performance and on how the user's body impacts other phone signal operations, specifically GSM signaling.

\subsection{GPS Operation Principles}

GPS satellites send signals for civilian use at the L1 frequency at $1.575 \mathrm{GHz}$; these signals are modulated with a Pseudo-Random Noise (PRN) code unique to each satellite. A GPS receiver tries to acquire each GPS satellite's signal by correlating the signal spectrum it receives at L1 with a local copy of the satellite's PRN code. An acquisition is successful, once the local copy is in sync with the received signal, which requires shifting the copy appropriately both in time and in frequency. The latter shift is due to the Doppler effect caused by the satellite's and the user's relative motion. Once a satellite's signal has been acquired, the receiver tracks it, that is, the receiver continuously checks the validity of the shift parameters above and updates them if necessary.

Each satellite's signal is modulated not only with its PRN code but additionally with a navigation message, which contains almanac data (for easier acquisition of further satellites) as well as its precise ephemeris data, that is the satellite's predicted trajectory as a function of time, allowing GPS receivers to estimate the current position of the satellite. Finally, to achieve precise 3D positioning with a standard GPS receiver via trilateration, the positions of and distances to at least 4 satellites have to be known; those distances can be computed from the time shift maintained while tracking the respective satellites. As a general rule, the more satellites can be tracked, and the wider they are spread over the sky as seen by the user, the more precise the positioning -due to the additional distance data and a satellite geometry resulting in less error-prone lateration.

A popular enhancement of GPS positioning is given by Assisted GPS (A-GPS) [25], which provides assistance data to GPS receivers via an additional communication channel, which for in-phone GPS operation is usually the cellular network. This assisting data contains ephemerides and often also atmospheric corrections. A-GPS eases satellite acquisition and can therefore drastically reduce the time to first fix and the initial positioning imprecision of a receiver, once the assisting data has been transmitted. Furthermore, A-GPS can improve positioning accuracy by eliminating systemic, e.g. atmospheric, error sources [16, Chapter 13.4].

\subsection{Environment Impacts on GPS Performance}

GPS performance degrades in terms of both coverage and accuracy when experiencing problematic signal conditions, e.g. in urban canyons and especially in indoor environments. The cause for this is termed signal fading, subsuming two fundamental signal 
processing obstacles: First, when GPS signals penetrate building materials, they are subjected to attenuation, resulting in lower signal-to-noise ratio (SNR). Furthermore, the signal is subject to multipath phenomena: Reflection and refraction of the signal results in multiple echoes of the line-of-sight (LOS) signal arriving at the receiver. Low signal-to-noise ratios and multipath handicap both acquiring and tracking GPS signals and usually result in less reliable positioning due to less suitable satellite geometry and less accurate individual time shifts measurements. For investigations of GPS positioning in urban and indoor environments and its limitations, see, e.g., [82227]. High-Sensitivity GPS (HSGPS) [12] subsumes advances in GPS receiver technology to alleviate the limitations mentioned above. HSGPS is claimed to allow tracking for received GPS signal strengths down to $-190 \mathrm{dBW}$, corresponding to a nominal SNR value of $14 \mathrm{~dB}$ : three orders of magnitude less than the GPS signal strength to be expected in open-sky conditions [16]. These thresholds are constantly being improved using new processing techniques [25, Ch. 6].

\subsection{In-Phone Signal Recption and Antenna Design Considerations}

Today, most smart-phones allow for reliable and accurate GPS positioning in open-sky conditions. Van Diggelen lists the main technological advances which have led to this achievement, stating furthermore, that "we thought the main benefit of this would be indoor GPS, but perhaps even more importantly it has meant very, very cheap antennas in mobile phones" [26]. It is agreed within the GPS research community, that antenna design, placement, and utilization is key for the further improvement of in-phone GPS positioning [5]6]. Central aspects in this challenge are the cost-effectiveness of the antenna design and the limiting of interference caused by other in-phone components, such as the GSM communication module. Finally, increasing form factor minimization also increases the constraints on antenna size, suggesting cohabitation, i.e., the use of one antenna for multiple services such as GPS reception, and GSM, Bluetooth, or WiFi communication [5]. More recently, a growing focus on in phone GPS technology lies on limiting the power consumption [25] and consequently, most GPS chip manufacturers emphasize and provide details about the improved energy-efficiency of their latest products for in-phone integration.

While the in-phone GPS reception is strongly influenced by the kind of environment, e.g. urban or indoor, another source of impairment can be the user himself, more specifically the parts of the user's body, which are either i) close to or even ii) contacting with the GPS in-phone antenna, or iii) just blocking the line-of-sight between the antenna and specific GPS satellites. All these three phenomena have impacts on GPS reception, the magnitude of which depends also on the design of the smartphone used. Sokova and Forssell give indications, that in difficult positioning conditions, e.g., indoor environments even pedestrians passing by can cause severe impairment of GPS reception [21]. In general, the closer the body is to a receiving antenna and the more it shields it, the more signal power dissipates into the body, impairing the desired resonation of the antenna with the incoming signal. Such body effects have been investigated thoroughly for the sending and receiving of signals of various types, most prominently for cell phone communication signals [1]2]. The above research identified that for the quality of signal sending and reception the following (interrelated) parameters are crucial: 
the antenna type, its location within the phone, and the way the user holds the phone, specifically the phone's orientation and the amount of body shielding and contact the phone's antenna is subjected to.

Furthermore, the results of these studies allow the conclusion that the body impacts on signal reception are complex to model in simulation and that the respective results often differ from the effects as observed in real-world situations. Furthermore, the effects depend in a complex manner on the signal frequency. Consequently, these studies provide an intuition about the body effects on the reception of signals at the GPS signal frequencies -but due to the GPS signals differing from GSM signals not only in frequency, but also in strength, purpose and structure, these studies don't allow for proper predictions of body effects on GPS reception in real-life use-cases, and even less so for predictions of the resulting impacts on the experienced GPS positioning performance.

\section{Quantifying the Body and Phone Impact on GPS Reception}

In this section we present results from measurements designed to quantify the impact of the user's presence and handling of the phone in real-world settings. More specifically, we measure impacts of various grip styles selected according to previous work; both in this and the subsequent section we will relate these impacts also to effects originating from the user's environment.

\subsection{Methodology}

The primary measure we used in our analysis of in-phone GPS performance are the signal strengths as they are experienced on the phones for the GPS satellites tracked by the phone. It has been observed that this set of signal strength values gives a good indication of overall GPS positioning quality including the essential performance parameters availability and accuracy, and we provide evidence for that in Section 3.2. Hence, to evaluate the impairment of GPS performance caused by a form of user interaction, e.g., a certain grip style, we measured the signal strengths in respective setups and compared the observed SNR values to those observed on a reference phone affected neither by body nor user environment impacts. To be able to draw valid conclusions from such signal strength comparisons, it is essential, that the everyday difference in observed signal strengths between two unaffected reference phones is small. To validate this assumption, we have collected sixty hours of measurements with two unaffected Google Nexus One phones placed statically in open-sky conditions, 2 meters apart from each other, with no nearby pedestrians, and running only our measurement collection software. As we measure body and user environment impacts over 10 minutes using two phones and average the GPS signal reception properties over this time span, the two assumptions we depend on are that a) the deviation in signal strength between phones within the ten minutes are small and b) that for the same phone the signal properties measured differ only slightly in consecutive measurements. Mainly to validate our measurement setups, we also investigated whether interference between close by GPS receivers [5] can impair any GPS performance measures: In several experiments in which several GPS enabled phones were placed as close as 5 centimeters apart we observed no visible degradations. 
Table 1. Absolute signal strength deviations between two unaffected phones in open sky conditions for Google Nexus One phones

\begin{tabular}{lllllll}
\hline Scenario & \multicolumn{3}{c}{ Four Strongest Satellites $[d B]$} & \multicolumn{3}{c}{ All Satellites [dB] } \\
& $\mu$ & $\sigma$ & $\max$ & $\mu$ & $\sigma$ & $\max$ \\
\hline Between Phones & 1.10 & 0.86 & 4.35 & 1.20 & 0.93 & 4.91 \\
Same Phone (Consecutive) & 0.62 & 0.47 & 2.29 & 1.01 & 0.81 & 4.04 \\
\hline
\end{tabular}

As shown in Table 1 for the validation of our assumption, we give quantifications of signal strength differences by mean, standard deviation and maximum differences averaged over i) the four GPS satellite signals which are received strongest on the phone and ii) over all satellite signals tracked by the phone 1 . The results in Table 1 indicate mean variations around $1 \mathrm{~dB}$ with standard deviations below $1 \mathrm{~dB}$ and maximum variations below $5 \mathrm{~dB}$. It follows that for our results to deviate from the mean with more than one standard deviation (this deviation is relevant as visual inspection of the distributions supports that they are normally distributed), the signal strength would have to differ at least $1.96 \mathrm{~dB}$ in the case of the four strongest satellites and 2.13 for all satellites.

\subsection{Measured Impacts of the User Body on GPS Reception}

As reviewed in Section 2 there are results from studies of, e.g., cellular technologies that confirms that bodies negatively impact signal reception in mobile phones. We want to add to this knowledge by studying the effects on the GPS antenna. In this specific study we focus primarily on the Google Nexus One phone but also present results for the Nokia N97.

To select relevant hand and body placements we base our selection on a study by Pelosi et al. [19] who identified common hand grip styles for both data and talk mobile phone usage. Based on their study we have selected three data style grips one with 3 fingers in the bottom third of the device, a five finger style and a double hand style, and a soft and a firm talk style grip with five fingers, as depicted in Figure 1. As GPS usage is also relevant when the user does not have the phone in the hand we have also evaluated an overarm jacket pocket placement, e.g., similar to popular overarm straps for runners, a trouser pocket placement and a placement in the top of a bag carried by a person. To limit the study we did not consider special casings of the phone or special phone configurations, e.g., opening of the keyboard on the Nokia N97. We conducted the experiments outdoors in open sky conditions and collected measurements for 10 minutes with one affected phone held in the evaluated body position and a unaffected phone statically placed 1.5 meter away from the person carrying out the experiment. To compare the data we calculate the drop in signal strength as the mean signal strength difference between the measurements from the affected and the unaffected phone.

The results listed in Table 2 from the measurements with two Google Nexus One phones show that signal strength drops depending on the hand grip style and body

\footnotetext{
${ }^{1}$ We chose to give measure - i) additional to measure - ii) because the strongest satellites will also be the most important contributors to the positioning accuracy of the GPS. Therefore, a significant drop of their SNR will have a higher impact than that of the SNR of already weaker satellites that the GPS may weigh less (or not even consider) in the position computation [10].
} 


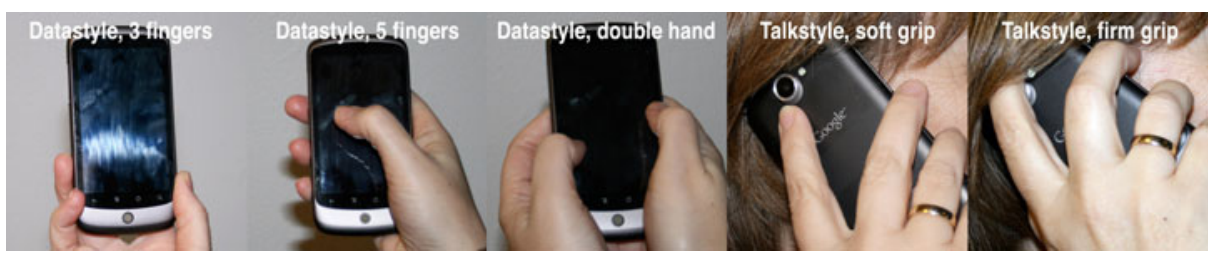

Fig. 1. Illustrations of different hand grip styles

placement. The most significant drops between 6.9 to $10.6 \mathrm{~dB}$ are experienced with Talkstyle, firm grip, 5 fingers and Datastyle, double hand. For comparison, Kjærgaard et al. [8] lists the following values for attenuation of glass $2.43 \mathrm{~dB}$, a wooden wall 4.8 $\mathrm{dB}$, a brick wall $10.38 \mathrm{~dB}$ and reinforced concrete $16.7 \mathrm{~dB}$. Generally the drops for the four strongest and all visible satellites correlate only the Running style, overarm jacket pocket scenario deviates where the drop considering all satellites is $3.7 \mathrm{~dB}$.

Table 2. Drops in signal strength for the four strongest and all satellites with different hand grip styles and body placements comparing pairs of Google Nexus One and Nokia N97 phones

\begin{tabular}{lcccc}
\hline & \multicolumn{2}{c}{ Google Nexus One } & \multicolumn{2}{c}{ Nokia N97 } \\
Scenario & Four Strongest $[d B]$ & All $[d B]$ & Four & Strongest $[d B]$ All $[d B]$ \\
\hline Running style, overarm & 0.3 & 3.7 & - & - \\
Datastyle, 3 fingers & 0.4 & 0.4 & 4.7 & 2.7 \\
Everyday style, bagpack & 1.5 & 2.0 & - & - \\
Talkstyle, soft grip, 5 fingers & 2.5 & 2.0 & - & - \\
Talkstyle, firm grip, 5 fingers & 6.9 & 7.3 & 17.3 & 14.2 \\
Datastyle, 5 fingers & 8.0 & 3.6 & 11.8 & 10.5 \\
Everyday style, trouser back pocket & 9.4 & 6.6 & - & - \\
Datastyle, double hand & 10.6 & 8.8 & 16.1 & 14.5 \\
\hline
\end{tabular}

To argue that the signal strength drops are not only pertinent to the Google Nexus One phone we collected similar measurements with two Nokia N97 phones for a subset of the scenarios. The results are also listed in Table 2 and indicates even bigger drops in the range of $4.7 \mathrm{~dB}$ to $17.3 \mathrm{~dB}$ which is five $\mathrm{dB}$ higher than for the newer Google Nexus One for similar hand grip styles and body placements. From these measurements we can conclude that the body impact is present and in some cases amounts to the attenuation experienced in indoor environments.

To quantify the effect on positioning accuracy during everyday use measurements were collected by a person walking a 4.85 kilometer tour twice through both opensky and urban positioning conditions carrying six phones with different placement. The data set consists of ground truth positions and $1 \mathrm{~Hz}$ GPS from the built-in sensors in the Google Nexus One and the Nokia N97. The ground truth was collected at $4 \mathrm{~Hz}$ with a high accuracy u-blox LEA-5H receiver with an dedicated antenna placed on the top of a backpack carried by the collector. The ground truth measurements were manually inspected to make sure they followed the correct route of the target. 
Figure 2 shows error plots for four of the Google Nexus One and Nokia N97 traces, respectively. The error is computed as the distance between the ground truth positions reported by the dedicated GPS to the positions reported by the phones. The figures depict cumulative distributions of the individual positioning errors throughout the traces. From the figures one can observe a significant difference in accuracy comparing the nearly body unaffected placements of Upper compartment of bagpack and Datastyle, 3 fingers to the affected trouser placements and the Datastyle, 5 fingers. Considering the median, the increase in error is for the Google Nexus One from five meters to ten meters and for the Nokia N97 from ten meters to twenty meters. One can therefore conclude that the body impact can have a strong impact on the positioning accuracy. In a study, conducted at the same time as ours and for three mobile phones Vaitl et al. [24] also identified the phone placement within trouser pockets as the worst for GPS accuracy.
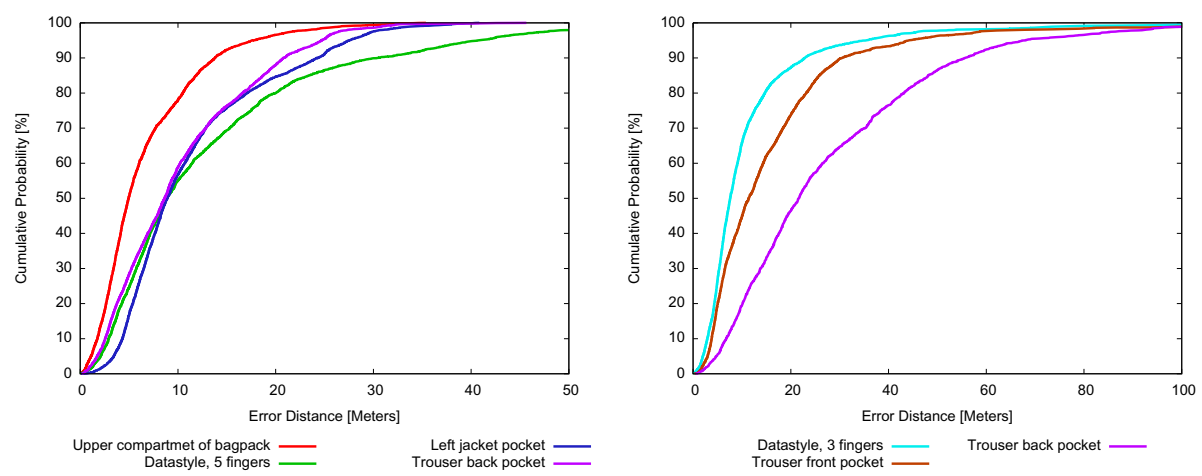

Fig. 2. Drops in positioning accuracy with different hand grip styles and body placements for Google Nexus One (left), and Nokia N97 (right)

The body effects also impact the positioning availability in the everyday measurements, but only for the Nokia N97, whereas for the Google Nexus One, which was released in early 2010, 16 months after the N97, there were no major drops. For the N97s the availability dropped from $88 \%$ during collection in the Datastyle, 3 fingers case to $51 \%$ for the back pocket trouser placement. Availability drops may occur also on the Nexus One, as we observed in the data set collected to evaluate the method proposed in Section 4. In four urban and five indoor data traces a Nexus One placed in a trouser pocket did not produce any fixes at all and in one urban and four indoor data traces, and a Nexus One held with the Datastyle, 5 fingers grip style did produce only very few. In the same traces both a phone held with grip style Datastyle, 3 fingers and a reference phone placed some meters away from the person collecting the data produced continuous fixes throughout the experiments. Therefore, we can conclude that body effects impact GPS availability, however, more significantly for the N97 than for the Nexus One. 


\section{Sensing and Classifying Impairment Sources}

To assist both application systems as well as the user in understanding and mitigating body and environment-induced effects, we aim to provide information about which effects are currently impacting the GPS device's performance and to which extent. In the following, we present an approach for differentiating such effects and respective sources utilizing only GPS signal quality data, in particular SNR measurements paired with directional information about GPS satellites; note, that such data is made available by most popular last generation smart-phones. The information about sources of GPS performance impairment, that we provide, adds to existing user assistance such as radar views of satellites strengths and accuracy estimates, and can be delivered as either visual, audible or tactile feedback, assisting, e.g. in answering questions such as "What is impacting my positioning accuracy?" and "Can I improve GPS performance by changing my grip style or placement?".

\subsection{Classification Concept and Procedure}

The proposed concept is illustrated in Figure 3. An in-phone GPS module outputs signal quality measurements -even in conditions in which only few and very weak signals can be acquired. Therefore, our method is functional even in cases where the GPS module is not able to produce any position fixes at all. From the signal quality measurements a range of features are computed with the help of an open-sky model that estimates how strong signals would be received for a given satellite and on the device if placed in open-sky conditions and not suffering from body, indoor or urban effects.

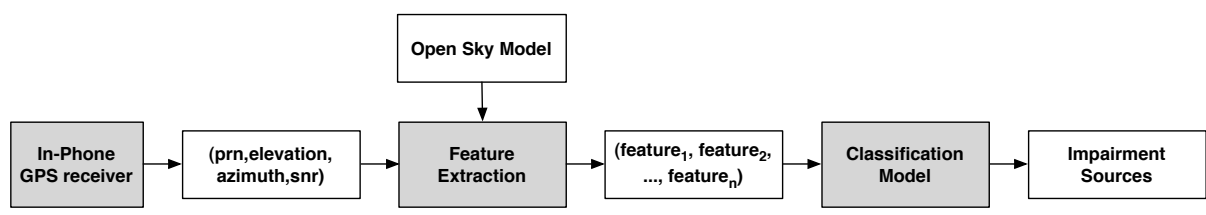

Fig. 3. Process for classifying impairment sources via analyzing in-phone signal measurements

Classification output domain. The classification model outputs information about inferred sources of GPS performance impairment. We have chosen to consider two types of GPS positioning impairments, environment- and body-induced ones. Furthermore, we considered two resulting categorizations: In the first one we distinguish twelve categories, i.e. twelve potential outcomes of the classification, corresponding to the twelve combinations of three environment types (open-sky, urban and indoor) and four phone placement and posture types (datastyle 3 fingers, datastyle 5 fingers, trouser back pocket, and no-body reference). In the coarser second categorization, we consider only six categories, combining the three environment types (open-sky, urban and indoor) with cases of no or weak body effects (datastyle 3 fingers and no-body reference) and cases of significant body effects (datastyle 5 fingers and trouser back pocket). 


\subsection{Feature Extraction}

The aim in designing suitable features for classification is to numerically capture the occurrence of patterns which -ideally-are specific to a single environment or on-body phone placement or posture, or -more generally- allow us to separate different (combinations of) environment and body placement types. In the following, we first sketch some of these patterns we look for, then we present features designed for detecting them, after introducing a utilized reference model, which holds expected SNR values in open-sky conditions. Some of the patterns we are looking for include:

When separating environment types. Naturally, for open-sky environments hardly any SNR drops or deviations w.r.t the model of SNRs in open-sky conditions occur. If deviations exist, they are distributed normally over both azimuths and elevations of the satellites tracked. In urban environments, drops occur for low elevation satellites, while the signals of higher satellites are received stronger. Indoors, satellite signals are received strongest through windows and wall openings, i.e. from satellites at low elevation and within 'horizontal clusters', i.e. at specific azimuth ranges, corresponding to window areas and wall openings.

When separating phone placement types. The blocking effect of the user body shows for different on-body placements in SNR drops of a particular range of azimuth values: E.g., for the trouser back pocket-placement this range is almost hemispherical. In contrast, when the user holds the phone in hand, the attenuation is more evenly distributed with regards to azimuth. Furthermore, different grips styles can often be distinguished by the overall amount of attenuation.

An Empirical Model of Open-Sky Conditions. Our identification of GPS reception impairments is based mainly on interpreting signal degradations. An indicator of the latter, which is even more suitable than the absolute SNR values recorded, is given by the drops of SNR w.r.t. ideal conditions, i.e. when not impacted by body- or environmentinduced effects. Therefore, our system is supported by an Open Sky Model which provides estimations of the SNR values to be currently expected on the device at the user's geographic position.

To the best of our knowledge, there does not yet exist an accurate theoretical model for open-sky GPS signal conditions. There are two main reasons for this, firstly, that SNR values depend both on properties of the antenna and the receiver chip, and secondly, that the transmission power of GPS satellites vary depending on their generation and age. Therefore, to characterize open-sky conditions we propose to use a devicespecific and empirical parametrized model. This model holds for each GPS satellite a function which maps for each GPS satellite its evaluation to a Gaussian distribution of the SNR of that satellite at that elevation, as recorded by the device. The resulting function table contains less than 3000 entries, .i.e. a mere 60 kilobytes. The motivation for modeling not only average SNR values, but also error distributions is that deviations are caused by several error sources, such as atmospheric weather, ground multi-path effects and integer rounding imprecision of the elevation data. Note, that since GPS orbits repeat every sidereal day, these differences are observable from the mappings which each use only 24 hours of the recorded data. The daily SNR pattern, as well as 


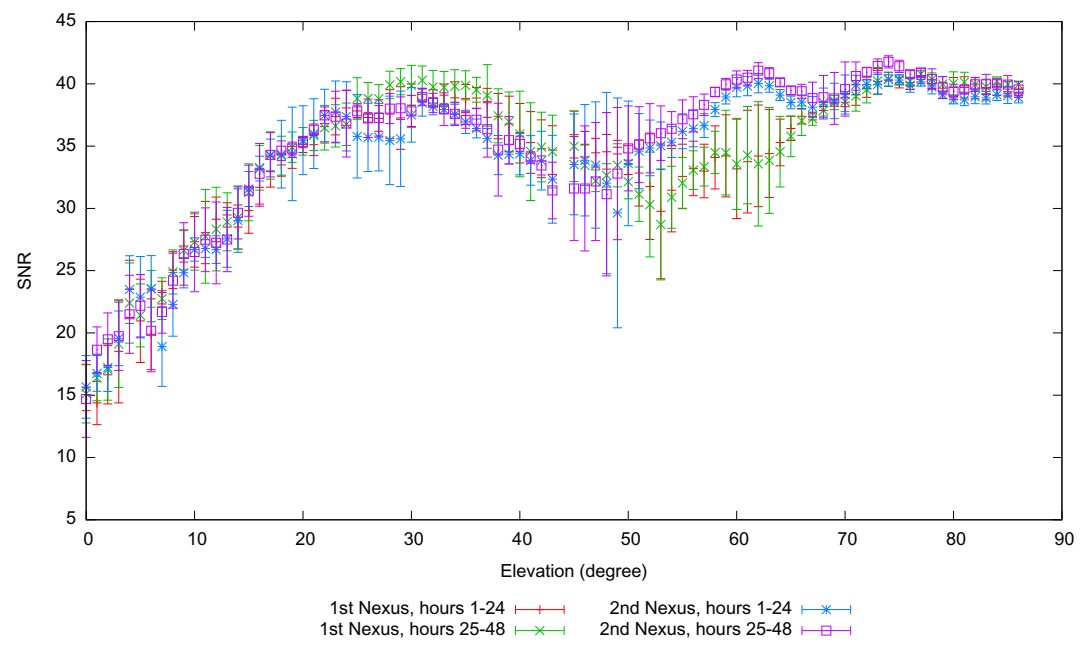

Fig. 4. SNR measurements for GPS satellite PRN 31, recorded on Google Nexuses in open-sky no-body conditions

differences in this pattern observed over two consecutive days are depicted exemplary for GPS satellite PRN 31 as recorded in Aarhus, Denmark, in Figure 4.

As our model's mappings are gained by empirical data won over 48 hours at one particular location, the accuracy of the model diminishes with the temporal and spatial distance from that recording. We will though later on present evidence that our system performs well also with less accurate or less detailed models.

Classification Features Employed. We now give an overview of the 29 features our current classification is based on, as well as discuss their suitability and limits in providing a successful classification of (combinations of) body-induced and environmentinduced impairments.

Features Based on Averaged SNR Drop: One of the features used in classification considers the experienced SNR drop w.r.t. the open sky model, averaged over all satellites within the GPS constellation which were trackable according to the open sky model. This feature captures the overall level of signal attenuation experienced. Figure 5(a) illustrates the differentiation of phone postures and placements achievable by this feature: The probability distributions shown represent output of the feature, i.e. average SNR drops. Each distribution subsumes data, described in more detail in Section 4.3. from 12 five-minute measurements for each particular combination of environment and phone-body context. Note, that to achieve better visualization of the characteristica of the distribution, feature output was beforehand mapped to bins. The plot shows, that in open-sky environments almost no SNR drops occur for the grip style using only 3 fingers, while in contrast large drops occur (in any given environment), when the phone is held firmly with 5 fingers, and even significantly larger drops occur when the phone is kept in the back pocket of a trouser. Furthermore, the two distributions in Figure 5(a) showing data gathered in urban settings, show that the environmental 


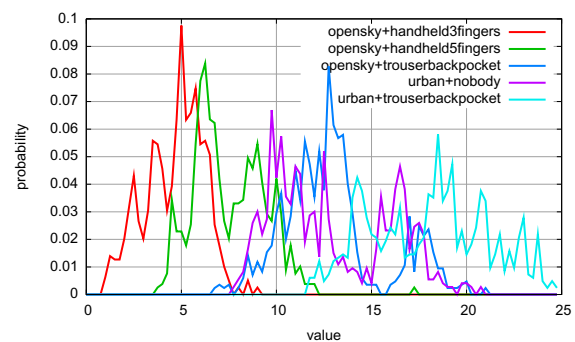

(a) SNR drop w.r.t. opensky model, averaged (b) over all receivable satellites

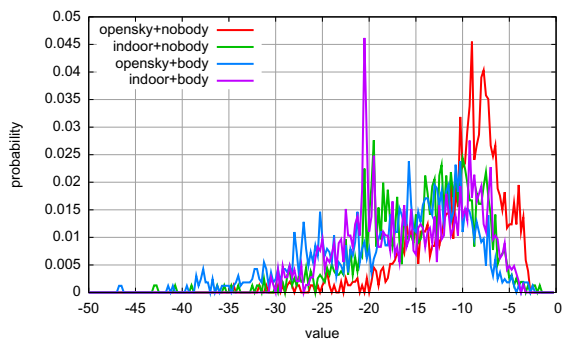

(b) Maximal SNR difference between two satellites, consecutive in the increasing order of the received satellites by elevation

Fig. 5. Probability plots exemplifying two classification features

attenuation visibly 'blurrs' the separation between different contexts; a fact that represents a general challenge for the body context classification: As a rule of thumb, the more attenuating and impairing the user's current environment is in itself, the harder it becomes to differentiate between different phone-body contexts.

Features Based on Elevation Order: The following feature is designed to distinguish environment types: It captures the maximally negative difference of SNR between two satellites, consecutive in the increasing order of the received satellites by elevation. Figure 5(b) shows output of this feature, originating from the measurements mentioned above. The distributions shown result from grouping measurements, undertaken in open-sky as well as in indoor areas and with various body-phone contexts, by severity of GPS impairment -following the coarser one of the categorizations described in Section 4.1. The plot shows, that for most indoor locations the feature output values are high. This is because satellites are received most strongly at low elevations, e.g. through windows, and because these satellites are ultimately followed in the elevation ordered sequence by a satellite that is highly attenuated by either walls or ceilings. In contrast, the open-sky measurements provide feature values close to zero. This again gives evidence, that if the impairment in one domain -in this case the body-induced one- is severe, it becomes harder for the feature to differentiate impairments of another domain -in this case of the user environment, i.e. to tell apart indoor locations from open-sky ones. Another feature is obtained as a variant of the one just described: It measures the maximal positive, instead of negative, SNR difference; this allows for identifying the urban environment type, since in urban canyons usually the satellites above the 'skyline' of surrounding buildings are received significantly stronger.

Features Based on SNR Drop Order: Three of the features currently employed are computed on basis of the sequence of the tracked satellites, sorted by the SNR drop experienced for them. E.g., when averaging over the azimuth difference between satellites consecutive in that sequence, the resulting feature captures the entropy for the sequence w.r.t. azimuth; for open-sky no-body conditions this entropy is usually high, while the lowest entropy was obtained in a no-body setup in indoor locations: Satellites similar in 
azimuth often reach the user through the same building element and therefore exhibit also similar SNR values.

Features Based on Azimuth Order: Alternatively, if one sorts the satellites instead by azimuth, one can capture, e.g. hemispherical, body shielding effects for in-pocket setups: One of our features identifies the azimuth half-space separation, for which the difference between the averaged signal drop in the respective hemispheres is maximized. This feature naturally generalizes for angles other than 180 degree.

Further Features: Further features are extracted from already established signal quality indicators, e.g., DOP values as provided by the phone's GPS system [16]. Feature design techniques, which we consider worth of exploring in future work, we discuss in Section 5 .

\subsection{Classification Results}

As depicted in the procedural outline given in Figure 3, the classification model has to infer present GPS positioning impairments, once provided with feature outputs, computed as described in the previous section. To implement the classification model we chose to use the Weka Machine Learning toolkit [28]. Prior to processing the feature outputs, we aggregate them by averaging over a five second window to remove outliers2.

To evaluate the proposed classification concept, we collected a data set at three opensky, three urban, and three indoor exemplary locations, employing different phone-body contexts in order to cover all twelve classification context categories listed in Section 4.1. At each location four consecutive measurements were collected at a fixed position, and for two opposite directions e.g. facing north and south, resp. For each location and orientation, measurements were undertaken by two users of differing stature in order to investigate the influence of body physique on the GPS reception and on our impairment classification process. In each measurement, four Google Nexus One phones were used where one was placed 2 meters away as reference, one was placed in the user's trouser back pocket and the two remaining ones were held by the user with the Datastyle, 3 fingers and the Datastyle, 5 fingers grip style, resp. In total, 144 measurement traces of five minutes each were collected. Each trace contains GPS position fixes and signal quality measurements sampled at $1 \mathrm{~Hz}$. This experimental setup was designed to collect a balanced data set w.r.t. locations, users, orientations and body placement; however, since at some locations positioning availability was not $100 \%$, some categories naturally have fewer data samples. Thus, we have applied a re-sampling filter to balance the data, so that we are able to judge the performance of the classification model directly from the classification accuracies and confusion matrices.

In Table 3 we present classification results for six and twelve categories, resp., and for two different machine learning algorithms, the basic Naive Bayes algorithm and the more accurate $\mathrm{J} 48$ decision tree algorithm and for three types of evaluations: firstly,

\footnotetext{
${ }^{2}$ We evaluated different window sizes: For the window size chosen the classification results benefited from the resulting noise removal, whereas for larger windows it suffered too much from the size reduction of the data set.
} 
Table 3. Classification accuracy results for classifying GPS positioning impairments into six and twelve categories, resp.

\begin{tabular}{lccc}
\hline \multicolumn{4}{c}{ 10 Fold Cross-Validation Different Persons Different Orientation } \\
\hline 12 Categories & 59 & 44 & 46 \\
Naive Bayes & 94 & 50 & 51 \\
Decision Tree & & & \\
6 Categories & 73 & 71 & 66 \\
Naive Bayes & $\mathbf{9 6}$ & $\mathbf{7 5}$ & $\mathbf{7 3}$ \\
Decision Tree & & & \\
\hline
\end{tabular}

ten fold cross-validation on the complete data set, secondly, training with data for one person and testing the resulting classification model with data from the other person and, thirdly, training with data for the respectively first chosen orientation and testing with the data of the respective opposite orientation. The results show that the decision tree algorithm performs better than the naive Bayes algorithm with accuracy rates of $94 \%$ and $96 \%$ for twelve and six categories, respectively. However, there are indications of overfitting because separating the training and test data, either w.r.t. to person or orientation, lowers the results to $75 \%$ and $73 \%$, respectively, for six categories and even more for twelve categories. Similarly, training with data from only half of the investigated environments and subsequent testing with the remaining data, results in a lower classification accuracy -implicating, that for accurate classification in arbitrary environments training data from a broader variety of locations would be essential, as well as further development of the proposed features.

To further analyze how the errors are distributed, Table 4 shows the confusion matrix for the results of the decision tree algorithm with six categories and when separating training and test data w.r.t. the collecting person. From the matrix one can see that data from the classes open-sky, no body and urban, no body are classified highly accurate, whereas data from indoor, no body and indoor, body is not; the poor separation performance of the algorithm in this case shows in high confusion values of $28.9 \%$ and $30.8 \%$ between the two categories. This observation is in agreement with the statement made

Table 4. Confusion matrix for the decision three algorithms for six categories and with separate training and test data depending on the collecting person

\begin{tabular}{l|c|c|c|c|c|c|} 
& \multicolumn{7}{|c|}{ Classified As } \\
& $\begin{array}{c}\text { open-sky } \\
\text { no body }\end{array}$ & $\begin{array}{c}\text { urban } \\
\text { no body }\end{array}$ & $\begin{array}{c}\text { indoor } \\
\text { no body }\end{array}$ & $\begin{array}{c}\text { open-sky } \\
\text { nody }\end{array}$ & $\begin{array}{c}\text { urban } \\
\text { body }\end{array}$ & $\begin{array}{c}\text { indoor } \\
\text { body }\end{array}$ \\
\hline open-sky, no body & $\mathbf{9 2 . 1}$ & 0.1 & 1.2 & 6.6 & 0 & 0 \\
\hline urban, no body & 3.3 & $\mathbf{8 8 . 2}$ & 6.8 & 0 & 1.5 & 0.3 \\
\hline indoor, no body & 7.9 & 4.7 & $\mathbf{4 5 . 6}$ & 9.3 & 1.7 & 30.8 \\
\hline open-sky, body & 8.7 & 0 & 6.4 & $\mathbf{7 0 . 0}$ & 1.7 & 13.1 \\
\hline urban, body & 0 & 0 & 8.5 & 0 & $\mathbf{8 1 . 5}$ & 10.0 \\
\hline indoor, body & 0 & 0 & 28.9 & 0.2 & 0.9 & $\mathbf{7 0 . 0}$ \\
\hline
\end{tabular}


above, that in weak signal environments it is harder to differentiate body effects: even telling none body effects situations from those with body effects becomes challenging.

The results shown in Table 4 were obtained using an open-sky model that considered each satellite independently which has the drawback that reference data ideally should be collected at many places on the globe to account for local differences in observable signal strengths. Thus, we have also evaluated our method using an alternative, simpler model that combines gathered SNR data across satellites to compute the strength of GPS reception per elevation, averaged over all satellites. When using this model instead of the proposed open-sky model, the accuracy of the classifications, using either the naive Bayes or the decision tree algorithm, did only decrease by 2 to 3 percent.

\section{Discussion}

The work presented shows that classification of GPS impairment sources can be done, relying only on current GPS signal quality data obtainable on most last generation smart phones. In the following, we discuss the classification concept presented, how to utilize it, as well as future research directions for refining and improving it.

The feasibility of the classification concept in terms of classification accuracy has been documented in Section 4 . We expect further improvement from integrating additional features into our classification procedure. Among these will be the detection of geometric clusters (w.r.t. elevation and azimuth) of similarly strong satellites, to detect environmental features, such as window areas indoors, and street canyons in urban areas. Additionally, the consideration of the recent data history -additional to the most recent GPS signal quality data snapshot, may allow to more reliably detect and keep track of 'static' features such as windows, walls or buildings, and to tell them apart more easily from body features, which are always 'moving' with the user. Worth investigating is also the incorporation of indicators for the user's phone handling as well as his context, e.g., his transportation mode, which are provided through data from sensors other than the GPS. E.g., Vahdatpour et al. [23] propose to detect a device's on-body placement from accelerometer readings.

In terms of output semantics, an integration of further as well as more fine-grained classes of user environments, distinguishing between different building types and transportation vehicles, the user may currently be in, would be desirable, depending on user requirements and application scenarios. W.r.t. resulting classification accuracies, Table 4 illustrates the naturally poorer absolute accuracy when classifying into a higher number of classes; note, though that the majority of false classifications still determine the user environment correctly and only confuse similar phone postures and placements.

Furthermore, the issue that the diversity of the physiques of users result in drops in classification performance, as noted in Table 3, has to be addressed: First, the system should be trained through data gathered by subjects of various statures. Secondly, we plan to evaluate the benefits of providing the user with a training procedure, designed to determine the impact of the user's physique on GPS reception w.r.t., comparing the gathered data with the training data provided by users of various physiques.

The potential for application and middleware-specific benefits of the proposed on-device sensing and classifying of GPS impairments in real-time require further 
investigation, for improving both ad-hoc and general user behaviour, as well as for enhancing the positioning quality and quality awareness of GPS receivers. The latter can be achieved since identifying current impairment sources can inform the receiver which satellites' ranging data to trust: GPS receivers can ignore ranging information from individual satellites which are believed to be distorted or currently received only indirectly; while such selection has been shown to potentially improve GPS accuracy [11], knowing the current user environment is crucial for picking proper selection criteria: E.g., in open sky environments stronger signals usually provide preciser ranging information, whereas in indoor environments the contrary can hold, when the strongest signals are likely to be signal reflections, reaching the user through windows, but only indirectly and thus yielding large ranging distortations [8]. Furthermore, providing application developers with access to the classifications is an example of benefitial seamfull design for developers [14]: E.g., in a positioning middleware, which is designed following a seamfull design approach to provide translucency w.r.t. the positioning process, the classifications could be used as an input to adapt application logic.

To investigate to which extent the proposed classification can benefit ad-hoc and general user behaviour, we are currently considering a phone application which provides as feedback the classification results regarding reception impairments and sensed environments and phone postures. Additionally, we want to explore ways to provide visual or acoustic feedback, which not only assesses and classifies current GPS impairment sources, but which can also guide the user towards a more beneficial phone holding posture or placement, or help him identify more reception-beneficial spots within or close to his current environment, e.g., using information collected by fingerprinting GPS positioning quality [9]. Finally, the computational load and energy consumption induced by different impairment classification schemes should be investigated, to ensure that real-time processing is feasible on common smart phones -also for feature sets, larger than the currently used one. Our preliminary investigations indicate that real-time processing on common smart phones is possible for the presented system.

\section{Conclusions}

We presented a concept for sensing present impairments of GPS reception and positioning performance, and for classifying impairment sources in terms of body, urban and indoor context. Results obtained from a measurement campaign provided reasonable classification accuracy and a proof of concept, that both the type of environment, the user is currently in, as well as the way a user is currently holding or storing his phone can be determined with reasonable accuracy through analysis solely of GPS signal quality data as available on most modern smart phones. Finally, in Section 5 further improvements of the accuracy of the presented classification system were identified, and directions for how to bring benefits of such a classification concept to the users were illustrated.

Additionally, to assess user-body effects on GPS reception and to aid and inform existing and future research and application systems, we have empirically evaluated for different hand grip styles and body placements the respective effects on GPS positioning performance of modern GPS enabled smart-phones. The evaluation showed that GPS 
reception depends highly on how the phone is kept or held, and that body-effects can cause attenuation of average signal strength of up to $10-16 \mathrm{~dB}$, which is more than that caused by a typical brick wall, and can lead to a doubling of the median positioning error as experienced in open-sky conditions in the absence of body effects.

\section{Acknowledgment}

We thank Lasse Haugsted Rasmussen for his help in collecting measurements, acknowledge the financial support granted by the Danish National Advanced Technology Foundation for the project "Galileo: A Platform for Pervasive Positioning" (009-2007-2) and by the Danish National Research Foundation for MADALGO - Center for Massive Data Algorithmics and acknowledge Nokia and Google for hardware grants.

\section{References}

1. Al-Mously, S.I., Abousetta, M.M.: Anticipated impact of hand-hold position on the electromagnetic interaction of different antenna types/positions and a human in cellular communications. International Journal of Antennas and Propagation 2008 (2008)

2. Alexiou, A., Kostarakis, P., Christofilakis, V., Zervos, T., Alexandridis, A., Dangakis, K., Soras, C., Petrović, V., Kolundžija, B., Dordević, A.: Interaction between gsm handset helical antenna and user's head: Theoretical analysis and experimental results. The Environmentalist 25(2), 215-221 (2005)

3. Cai, Y., Xu, T.: Design, analysis, and implementation of a large-scale real-time locationbased information sharing system. In: Proceeding of the 6th International Conference on Mobile Systems, Applications, and Services, pp. 106-117. ACM, New York (2008)

4. Gaonkar, S., Li, J., Choudhury, R.R., Cox, L., Schmidt, A.: Micro-blog: sharing and querying content through mobile phones and social participation. In: Proc. of the 6th International Conference on Mobile Systems, Applications, and Services, pp. 174-186. ACM, New York (2008)

5. Haddrell, T., Ricquier, N., Phocas, M.: Mobile-phone gps antennas. GPS World 2 (2010)

6. Hurte, B., Leisten, O.: Getting into pockets and purses. GPS World 11 (2005)

7. A. Inc. Letter from apple regarding iphone 4. Press Release (July 2010)

8. Kjærgaard, M.B., Blunck, H., Godsk, T., Toftkjær, T., Christensen, D.L., Grønbæk, K.: Indoor positioning using GPS revisited. In: Floréen, P., Krüger, A., Spasojevic, M. (eds.) Pervasive Computing. LNCS, vol. 6030, pp. 38-56. Springer, Heidelberg (2010)

9. Kjærgaard, M.B., Weckemann, K.: PosQ: Unsupervised Fingerprinting and Visualization of GPS Positioning Quality. In: Proceedings of the Second International Conference on Mobile Computing, Applications, and Services (MobiCASE 2010). Springer, Heidelberg (2010)

10. Kuusniemi, H., Wieser, A., Lachapelle, G., Takala, J.: User-level reliability monitoring in urban personal satellite-navigation. IEEE Transactions on Aerospace and Electric Systems 43, 1305-1318 (2007)

11. Kuusniemi, H., Lachapelle, G.G.: GNSS signal reliability testing in urban and indoor environments. In: Proceedings of the NTM Conference (2004)

12. Lachapelle, G., Kuusniemi, H., Dao, D., MacGougan, G., Cannon, M.: HSGPS signal analysis and performance under various indoor conditions. Navigation, Inst. of Navigation 51(1), 29-43 (2004) 
13. LaMarca, A., Chawathe, Y., Consolvo, S., Hightower, J., Smith, I., Scott, J., Sohn, T., Howard, J., Hughes, J., Potter, F., Tabert, J., Powledge, P., Borriello, G., Schilit, B.: Place Lab: Device Positioning Using Radio Beacons in the Wild. In: Gellersen, H.-W., Want, R., Schmidt, A. (eds.) PERVASIVE 2005. LNCS, vol. 3468, pp. 116-133. Springer, Heidelberg (2005)

14. Langdal, J., Schougaard, K.R., Kjærgaard, M.B., Toftkjær, T.: PerPos: A translucent positioning middleware supporting adaptation of internal positioning processes. In: Gupta, I., Mascolo, C. (eds.) Middleware 2010. LNCS, vol. 6452, pp. 232-251. Springer, Heidelberg (2010)

15. Minamimoto, S., Fujii, S., Yamaguchi, H., Higashino, T.: Local Map Generation using Position and Communication History of Mobile Nodes. In: Proceedings of the 2010 IEEE International Conference on Pervasive Computing and Communications, pp. 2 -10 (2010)

16. Misra, P., Enge, P.: Global Positioning System: Signals, Measurements, and Performance, 2nd edn., Navtech (2006)

17. Mun, M., Reddy, S., Shilton, K., Yau, N., Burke, J., Estrin, D., Hansen, M., Howard, E., West, R., Boda, P.: Peir, the personal environmental impact report, as a platform for participatory sensing systems research. In: Proceedings of the 7th International Conference on Mobile Systems, Applications, and Services, pp. 55-68. ACM, New York (2009)

18. Kivekäs, T.L.O., Ollikainen, J., Vainikainen, P.: Bandwith, sar, and efficiency of internal mobile phone antennas. IEEE Trans. on Electromagnetic Compatibility 46(1), 71-86 (2004)

19. Pelosi, M., Franek, O., Knudsen, M., Christensen, M., Pedersen, G.: A grip study for talk and data modes in mobile phones. IEEE Transactions on Antennas and Propagation 57(4), 856-865 (2009)

20. Ryder, J., Longstaff, B., Reddy, S., Estrin, D.: Ambulation: A tool for monitoring mobility patterns over time using mobile phones. In: International Conference on Computational Science and Engineering, pp. 927 -931 (2009)

21. Sokolova, N., Forssell, B.: Moderate pedestrian traffic: Indoor hsgps receiver performance. European Journal of Navigation 5(3), 2-7 (2007)

22. Teuber, A., Paonni, M., Kropp, V., Hein, G.: Galileo signal fading in an indoor environment. In: Proc. 21st Intl. Techn. Meeting Satellite Division Inst. of Navigation (ION GNSS) (2008)

23. Vahdatpour, A., Amini, N., Sarrafzadeh, M.: On-body device localization for health and medical monitoring applications. In: Proceedings of the Ninth Annual IEEE International Conference on Pervasive Computing and Communications (2011)

24. Vaitl, C., Kunze, K., Lukowicz, P.: Does on-body location of a gps receiver matter? In: Int. Workshop on Wearable and Implantable Body Sensor Networks, pp. 219-221 (2010)

25. van Diggelen, F.: A-GPS: Assisted GPS, GNSS, and SBAS. Artech House, Boston (2009)

26. van Diggelen, F.: The smartphone revolution. GPS World 12 (2009)

27. Watson, R., Lachapelle, G., Klukas, R., Turunen, S., Pietil, S., Halivaara, I.: Investigating gps signals indoors with extreme high-sensitivity detection techniques. Navigation, Inst. of Navigation 52(4), 199-213 (2006)

28. Witten, I.H., Frank, E.: Data Mining: Practical machine learning tools and techniques, 2nd edn. Morgan Kaufmann, San Francisco (2005) 